\title{
Magnetostatic waves in a medium with damping
}

\author{
Pavel Makarov ${ }^{1, \star}$, Lidiya Maltceva ${ }^{1}$, Leonid Kotov ${ }^{1}$, and Vladimir Shcheglov ${ }^{2, \star \star}$ \\ ${ }^{1}$ Pitirim Sorokin Syktyvkar State University, Oktyabrsky 55, Syktyvkar, 167001, Russia \\ ${ }^{2}$ Institute of Radio-Engineering and Electronics of RAS, Mokhovaya 11-7, Moscow, 125009, Russia
}

\begin{abstract}
By means of consistent solution of the motion equation for magnetization the consideration of energy dissipation for magnetostatic surface waves propagating on in-plane magnetized ferrite plate is regarded. The complex character of the wave number caused by the dissipation is revealed. In this case the dispersion relations for the real and imaginary parts of the wave number are obtained. It is shown that dispersion curves are limited both by the wave number and frequency, and these restrictions tighten with an increase in the damping parameter. Also, it is predicted that there is a new set of branches of the backward dissipative waves. Both for forward and backward waves there is a critical value of the damping parameter and propagation angle, beyond which it does not exist.
\end{abstract}

\section{Introduction}

The utilization of the ferromagnetic structures for the magnonic devices is one of the perspective field of magnon spintronics and magnonics. Magnetostatic surface waves (MSSW) propagating in magnetic films are the basis for the construction of various microwave devices for analog and digital data processing, information transfer and control of $\mathrm{GHz}$ and $\mathrm{THz}$ dynamics [1-4]. Among the novel spin-wave based devices the magnonic coupler can also be considered as a candidate to beyond-CMOS devices [5, 6].

Dispersion is an inherent property of any waveguide based on ferrite. The signal attenuation level, the delay time, the phase shift and all other important characteristics dramatically depends on the dispersion law. Minimization of wave losses is a prerequisite for waveguide correct work, because the dissipation plays a cruicial role for the functionality of spin-wave devices. However, the MSSW damping is understudied as the vast majority of research is performed without consideration of attenuation $[4,7,8]$. Though, the damping may significantly change the dispersion law [9]. In particular, it limits the maximum value of the wave number, which results in increasing the minimum value of the signal delay time.

In large number of invesigations [3, 4, 7-9], propagation of MSSW is studied in the special cases when wave is transferred perpendicularly to the direction of the permanent external magnetic field or along it. However, the wave propagation at arbitrary angles is essential for magnonic networks circuits [10-12], multi-channel filters [1, 2], etc. Therefore, the aim of this research is to study the impact of the damping effect on the dispersion of MSSW at arbitrary angles.

\footnotetext{
^e-mail: makarovpa@syktsu.ru

$\star \star$ e-mail: vshcheg@cplire.ru
}

\section{Main equations}

The geometry of the problem is the following. An unbounded ferrite film of thickness $d$ is magnetized to saturation $4 \pi \mathbf{M}$ by the constant field $\mathbf{H}$. The Cartesian coordinates $O x y z$ are chosen so as the origin is at the middle of the film thickness, the $O y z$ plane is parallel to the plane of the film, and the axis $O x$ is perpendicular to latter. The $O z$ axis is oriented along the field $\mathbf{H}$. The wave vector $\mathbf{k}$ lies on the $O y z$ plane and is tilted by the angle $\varphi$ with respect to the axis $O y$.

It is assumed that the medium is homogeneous and isotropic. Waves are generated by a source with a predetermined frequency $\omega$. Thus, the wave amplitude can only be reduced if the wave number is the complex value: $k=\eta-i \xi$.

The Landau — Lifshitz equation [13] with the dissipative term of the Gilbert form is used to describe the motion of the magnetization vector $\mathbf{m}$ in a lossy medium (with the damping coefficient $\alpha$ ). In this geometry the dispersion equation for MSSW has the form:

$$
\beta-2 \mu \vartheta \operatorname{coth}(k d \vartheta)-1=0 .
$$

The equation above has been obtained by solving the Landau - Lifshitz - Gilbert equation with the boundary conditions. In (1) we have one constant parameter $d$, four variables: $k, \mu-$ the magnetic permeability of the medium of the form:

$$
\mu=1+\frac{\Omega_{H}+i \alpha \Omega}{\Omega_{H}^{2}-\left(1+\alpha^{2}\right) \Omega^{2}+i 2 \alpha \Omega \Omega_{H}},
$$

and two ancillary quantities $\vartheta$ and $\beta$, which are expressed by formulae

$$
\vartheta=\sqrt{\cos ^{2} \varphi-\frac{\sin ^{2} \varphi}{\mu}}, \quad \beta=\left(v^{2}-\mu^{2}+\mu\right) \cos ^{2} \varphi-\mu,
$$


where $v$ is the magnetic susceptibility of the medium having the form:

$$
v=\frac{\Omega}{\Omega_{H}^{2}-\left(1+\alpha^{2}\right) \Omega^{2}+i 2 \alpha \Omega \Omega_{H}} .
$$

The quantities $\Omega$ and $\Omega_{H}$ given in equations (2) and (4) are the normalized wave frequency and the magnetic field respectively:

$$
\Omega=\frac{\omega}{4 \pi \gamma M}, \quad \Omega_{H}=\frac{H}{4 \pi M},
$$

where $\gamma$ is the gyromagnetic ratio.

\section{Calculation results}

Figure 1 demonstrates the assemblage of dispersion curves for the real and imaginary parts of the wave number obtained by the numerical solution of equation (1). To construct figure 1 were used parameters for YIG-films: $\quad 4 \pi M=1750 \mathrm{Gs}, \quad \gamma=1.76 \cdot 10^{7} \mathrm{rad} /(\mathrm{s} \cdot \mathrm{Gs})$, $H=437.5 \mathrm{Oe}, d=15 \mu \mathrm{m}$.

We neglect the uniaxial anisotropy field $H_{u}$ in YIG due to its extreme smallness for film with thickness $d=15 \mu \mathrm{m}$, which may be considered as a bulk sample [14].

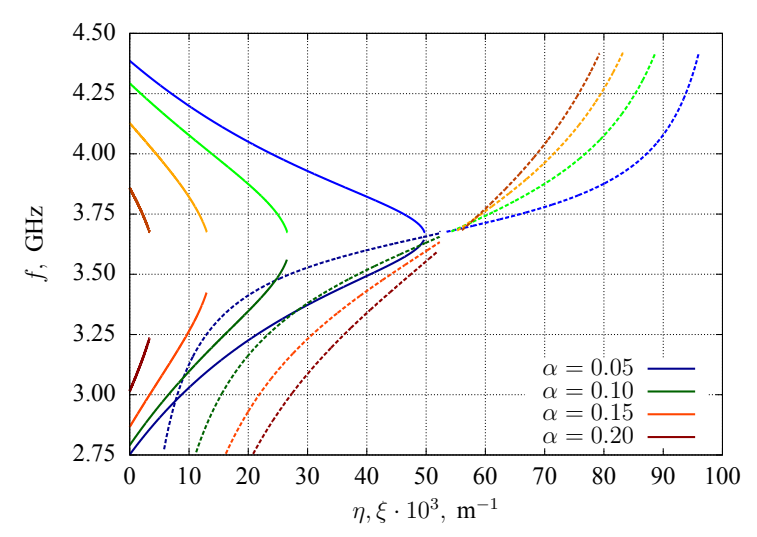

(a) $\varphi=0^{\circ}$

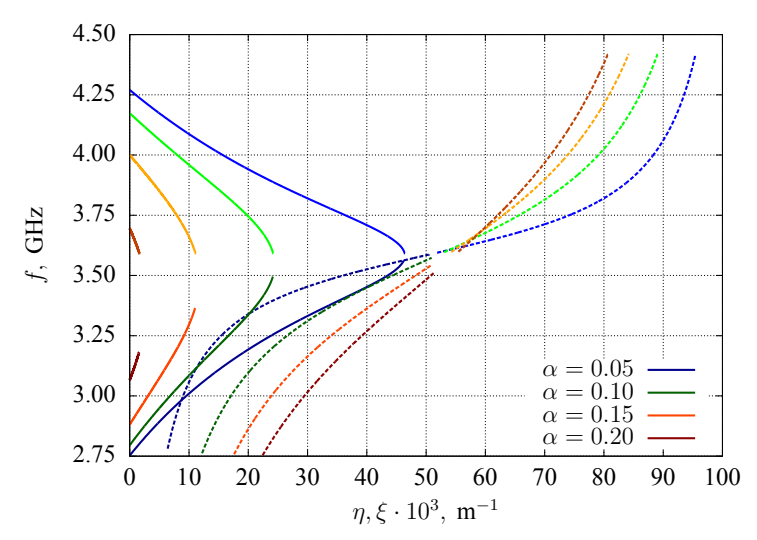

(b) $\varphi=15^{\circ}$

Figure 1. Dispersion law of the MSFSW and MSBSW with different values of $\alpha$ and $\varphi$. Solid curves $-f(\eta)$; dashed $-f(\xi)$.
Figure 1(a) illustrates the case when the wave propagates directly perpendicularly to magnetic field direction $\left(\varphi=0^{\circ}\right)$. A set of solid curves represents the dependence of the wave frequency from the real part of the wave number $f(\eta)$; the family of dashed curves displays the dependence of the frequency from the imaginary part of the wave number $f(\xi)$. Each of the four lines in the set is constructed for different values of the damping parameter $\alpha$. As figure 1(a) indicates, each set of curves is separated into two subset branches corresponding to dispersion of forward (MSFSW) and backward (MSBSW) waves.

Between the MSFSW and MSBSW branches of the dispersion curves $f(\eta)$ there is a bandgap of width $\Delta f$ that is the increasing function of the damping coefficient. For instance, with $\alpha$ ranging from 0.05 to $0.20 \Delta f$ increases from about $32 \mathrm{MHz}$ to $440 \mathrm{MHz}$. The frequency gap $\Delta f$ between forward and backward waves is associated with a change in the nature of the dispersion law from normal to anomalous. The transition between these two types of dispersion is associated with a slowing down of the energy transfer of the forward waves due to the dissipation.

Therewith, the frequency range of MSFSW and MSBSW significantly narrows with the increase of $\alpha$. Furthermore, there is a limitation to the maximum of $\eta$ for a given $\alpha$. For example, when $\alpha=0.05$, the value of $\eta_{\max }$ is approximately equal to $50 \cdot 10^{3} \mathrm{~m}^{-1}$, whereas when $\alpha=0.20, \eta_{\max } \approx 3.4 \cdot 10^{3} \mathrm{~m}^{-1}$ (figure $1(\mathrm{a})$ ). From the physical point of view, the reduction of the MSFSW and MSBSW frequency range with the increase of damping constant is related with the following feature. The speed of the wave energy transfer is reduced stronger with the growth of the dissipation. Therefore, the transition from the normal to the anomalous dispersion law occurs earlier both in terms of the wave number and frequency.

As we can see from the figure 1, for all given damping parameters, $\eta<50 \cdot 10^{3} \mathrm{~m}^{-1}$ (i.e., the wavelength $\lambda>125 \mu \mathrm{m}$ ). It is known, that the effect of the exchange interaction is noticeable only at a wavelength of $\lambda<1 \mu \mathrm{m}$ and in this case this leads to a change in the dispersion frequency by less than one thousandth of the normal frequency. Thus, under the conditions described in this paper, the effect of the exchange is negligible.

The dispersion curves for the dissipation $f(\xi)$ show that $\xi$ is a monotonically increasing function of the wave frequency, and with an increase of $\alpha$ this dependence becomes more linear. For this reason, the increase of $\alpha$ from 0.05 to 0.20 for MSFSW with frequency $3.1 \mathrm{GHz}$ leads to a growth of $\xi$ from $9.5 \cdot 10^{3} \mathrm{~m}^{-1}$ to $30.5 \cdot 10^{3} \mathrm{~m}^{-1}$.

Figure 1(b) displays the case of wave propagation in the direction of straight tilted by the angle $\varphi=15^{\circ}$ with respect to the axis $O y$. The basic regularities in this case are the same as for case $\varphi=0^{\circ}$ (figure 1(a)), however the restrictions of wave number and frequency are more enhanced.

Also, we obtained boundary frequences of magnetostatic waves by solving equation (1) in case when there is no damping. This frequencies, defined from the limit 
transition $\eta \longrightarrow 0$ and $\eta \longrightarrow \infty$ have the form:

$$
\left\{\begin{array}{l}
f_{1}=\gamma \sqrt{H(H+4 \pi M)}, \\
f_{2}=\gamma(H+2 \pi M), \\
f_{3}=\gamma \sqrt{(H+2 \pi M)^{2}+(2 \pi M)^{2}} .
\end{array}\right.
$$

Thus, the MSFSW propagate in a strictly defined frequency range $f_{1} \leqslant f \leqslant f_{2}$ and MSBSW in range from $f_{2}$ to $f_{3}$. The curves of the boundary frequencies as functions of a constant magnetic field are shown on figure 2(a). In addition, this figure shows the field dependences of width of the MSSW frequency range (dashed and dotted lines). The boundary frequencies of the MSSW are determined by the value of the saturation magnetization and can vary over a wide range.

On figure 2(b) we have dependence of the boundary frequencies of the magnetostatic waves, propagating in YIG film (saturation magnetization $4 \pi M=1750 \mathrm{Gs}$ ) on angle $\varphi$ when $\alpha=0$ and magnetic field $H=437.5$ Oe. We can see that higher frequency of forward and bacward surface waves is a deacreasing function of angle $\varphi$. Moreover, there is critical value of angle $\varphi$, above which surface waves cannot propagate.

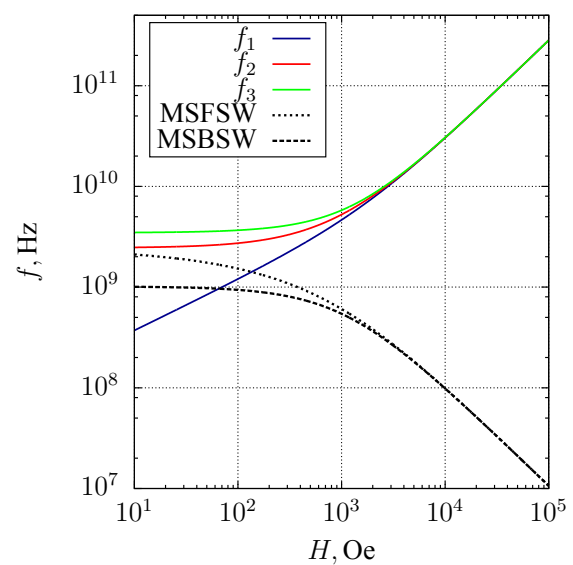

(a)

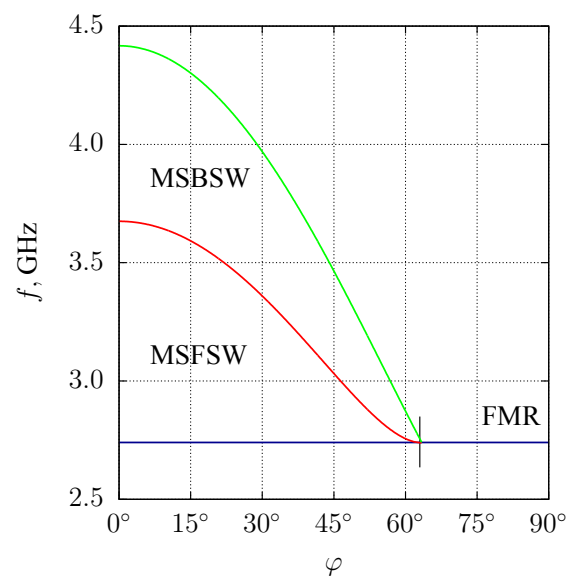

(b)

Figure 2. MSSW frequency spectrum without consider damping

\section{Conclusions}

In this study dispersion relations for the real and imaginary parts of the wave number were obtained. It was shown that dispersion curves are limited both by the wave number and frequency, and these restrictions tighten with an increase in the damping parameter.

A new branch of the backward dissipative waves was theoreticaly predicted. This solution doesn't contradict with the basic principles of electrodynamics, because it corresponds to the anomalous dispersion law.

MSFSW and MSBSW are characterised by existence of a critical value of the damping parameter and propagation angle, beyond which it does not exist. With the increase of the angle $\varphi$, limitation of dispersion curves tightens, the branches of the dispersion curves are shifted towards lower wave numbers and the damping parameter limits them from below and above.

The support from RFBR (grants \#17-02-01138, \#17-57-150001) are gratefully acknowledged.

\section{References}

[1] W.S. Ishak, Proc. of the IEEE 76 171-187 (1988)

[2] Y. Kajiwara et al., Nature 464 262-266 (2010)

[3] O. Kolokoltsev, N. Qureshi, E. Mejía-Uriarte, C.L. Ordóñez-Romero, J. Appl. Phys. 112013902 (2012)

[4] S. Jun, S.A. Nikitov, R. Marcelli, P. De Gasperis, J. Appl. Phys. 81, 1341 (1997)

[5] A.V. Sadovnikov et al. Appl. Phys. Lett. 107, 202405 (2015)

[6] A.V. Sadovnikov et al. Phys. Rev. Appl. 7, 014013 (2017)

[7] R.W. Damon, J.R. Eshbach, J. Phys. Chem. Solids 19 308-320 (1961)

[8] K.L. Wong et al., Appl. Phys. Lett. 105232403 (2014)

[9] J.H. Kwon, S.S. Mukherjee, P. Deorani, M. Hayashi, H. Yang Appl. Phys. A 111 369-378 (2013)

[10] V.E. Demidov et al. Sci. Rep. 5, 8578 (2015)

[11] A.V. Sadovnikov et al. Appl. Phys. Lett. 108, 172411 (2016)

[12] A.V. Sadovnikov et al. Phys. Rev. B 96, 060401(R) (2017)

[13] L.D. Landau, E.M. Lifshitz Statistical physics Part 2: Theory of the condensed state (Pergamon Press, Oxford, 1981) 387

[14] D. Stancil, A. Prabhakar Spin Waves. Theory and Applications (Springer, New York, 2009) 364 